\title{
Performing a Perinatal Autopsy
}

\author{
Sunil Jaiman ${ }^{1}$
}

Received: 18 September 2015/Accepted: 12 October 2015/Published online: 16 November 2015

(C) Society of Fetal Medicine 2015

\begin{abstract}
Intrauterine fetal demise or termination for malformation is adverse obstetric outcomes, agonizing for the parents and frustrating for health care providers. These events are often unpredictable and investigation entails not only the evaluation of the fetus/neonate but also the placenta. Though nothing can compensate the bereavement of families who have encountered fetal or a neonatal loss, a meticulous study of the placental, fetal, and neonatal tissues provides succor and help in the healing process. In recent times, with extensive advances in the pediatric specialty, the significance and requirements for perinatal autopsy have also commensurately heightened. There is an inordinate need for an accurate diagnosis in order to render genetic counseling and ascertain the implications of possible recurrence in future pregnancies. A conscientious perinatal autopsy, supplemented by cytogenetic and metabolic evaluation, followed by dissemination of the information to the parents, clinicians, and public health organizations is of paramount importance. This not only assists in the clinical management and bereavement closure but also helps in the reduction of perinatal mortality and morbidity. An autopsy protocol assists in performing an adequate perinatal dissection, facilitates the recognition and documentation of all relevant information and in addition also provides a format for the collection, recording and presenting of data which facilitates collaborative research. A brief account of the benefits and techniques of perinatal autopsy and the protocol are presented.
\end{abstract}

Sunil Jaiman

suniljaiman@gmail.com

1 Department of Anatomic and Perinatal Pathology and Cytology, Fernandez Hospital Unit 3, Plot 769, Road No. 44, Jubilee Hills, Hyderabad, Telangana State 500033, India
Keywords Perinatal autopsy · Protocol - Consent form · Autopsy technique · In situ examination · Provisional anatomic diagnosis

\section{Introduction}

The Autopsy Committee of the College of American Pathologists describes autopsy as "a medical-surgical procedure by a physician for the welfare of the living through the study of those patients for whom all our current knowledge and technology were inadequate" [1]. An array of brilliant protocols is available, though none of them can be deemed sacrosanct, and one may adopt/adapt any technique to salvage the maximum information for the benefit of the family and the clinician. For an erudite discussion of the plethora of existing protocols, the reader should refer to them [2-5].

The perinatal autopsy compilation here is brief and under the following sections:

1. Benefits of autopsy

2. Autopsy consent and permit

3. Radiographic examination

4. Photography

5. Instruments

6. Clinical and pre-autopsy considerations

7. Cytogenetic study

8. External examination

9. Incision

10. In situ examination and evisceration

11. Provisional anatomic diagnosis (PAD)

12. Placental examination

13. Microscopic examination

14. Final diagnosis

15. Classification of perinatal deaths 


\section{Benefits of Autopsy}

An autopsy helps to:

- Ascertain the cause of death

- Corroborate, supplement, or refute clinical diagnosis

- Evaluate diagnosis and therapy including new modalities

- Identify rare diseases

- Detect and discover new pathologic entities

- Document hitherto unknown information on disease manifestations

- Document growth and development

- Provide risk estimates for future pregnancy

- Further the cause of research and education

- Facilitate the investigation of environmental, occupational, and lifestyle causes of diseases

- Improve accuracy and usefulness of biostatistics

- Provide epidemiologic data

- Provide organs for donation and study

- Bereavement closure

\section{Autopsy Consent and Permit}

An unsuccessful outcome of pregnancy is a major catastrophe for parents. Seeking consent for performing an autopsy from grieving parents can be difficult. Nevertheless, the peremptory first step with an autopsy is the signed consent form and autopsy permit perusal by the attending pathologist (Fig. 6) in the Appendix. Dissection should be deferred or abandoned without the requisite consent/permit to avoid unnecessary legal entanglements.

Special permissions (dissection and removal of eyes) should be obtained prior to dissection. Autopsy constraints imposed by family or legal restrictions need to be adhered to. The prosector/institute should also familiarize itself with the local laws pertaining to autopsy examination and disposal of the remains.

\section{Radiographic Examination}

Every fetus submitted for an autopsy should mandatorily have a roentgenogram. Many conditions, especially suspected skeletal dysplasias, cannot be diagnosed without an X-ray picture. An antero-posterior and lateral view of the entire fetus is essential. Digital radiographs or faxitrons may be used, former being preferred.

\section{Photography}

Photographic documentation (external and internal) of the fetus/infant is of paramount importance. The pictures must be focussed to depict the abnormal with adequate reference points within the frame. Photographs may:

- Serve as a record for posterity

- Help ascertain diagnosis of a malformation syndrome

- Depict and preserve anatomic relationships of visceral lesions which otherwise are destroyed after dissection and evisceration

- Assist in identifying tissues submitted for microscopic examination

- Serve as records for family bereavement closure and litigation purposes

- Assist with publication and research

\section{Instruments}

The perinatal postmortem requires a fully-equipped laboratory with abundant light and a camera with a good macro-function in order to photograph small fetuses and organs. Ophthalmic instruments are excellent for small dissections. The requirements include (Fig. 1):

- Charts providing normal weights and measurements for newborns

- Sterile syringes and needles

- Sterile forceps and scissors for culture and karyotype

- Mounted magnifying glass

- Dissecting board, absorbent paper towels

- Large scale and electronic digital scale

- Measuring tape, rulers, callipers

- Scalpel handles, blades, large knife

- Small- and medium-sized forceps with and without teeth

- Stout scissors, Metzenbaum scissors, and small scissors

- Fine probes and hemostats of varying sizes

\section{Clinical and Pre-autopsy Considerations}

Fetal development is, in part, dependent on maternal health and intrauterine environment. It is imperative that the prosector obtains all relevant clinical and family history prior to the procedure. Genetic syndromes must be identified, if present. Obstetric history, ultrasound reports, including those pertaining to prior pregnancies and/or fetal 
Fig. 1 Basic instruments used for perinatal autopsy

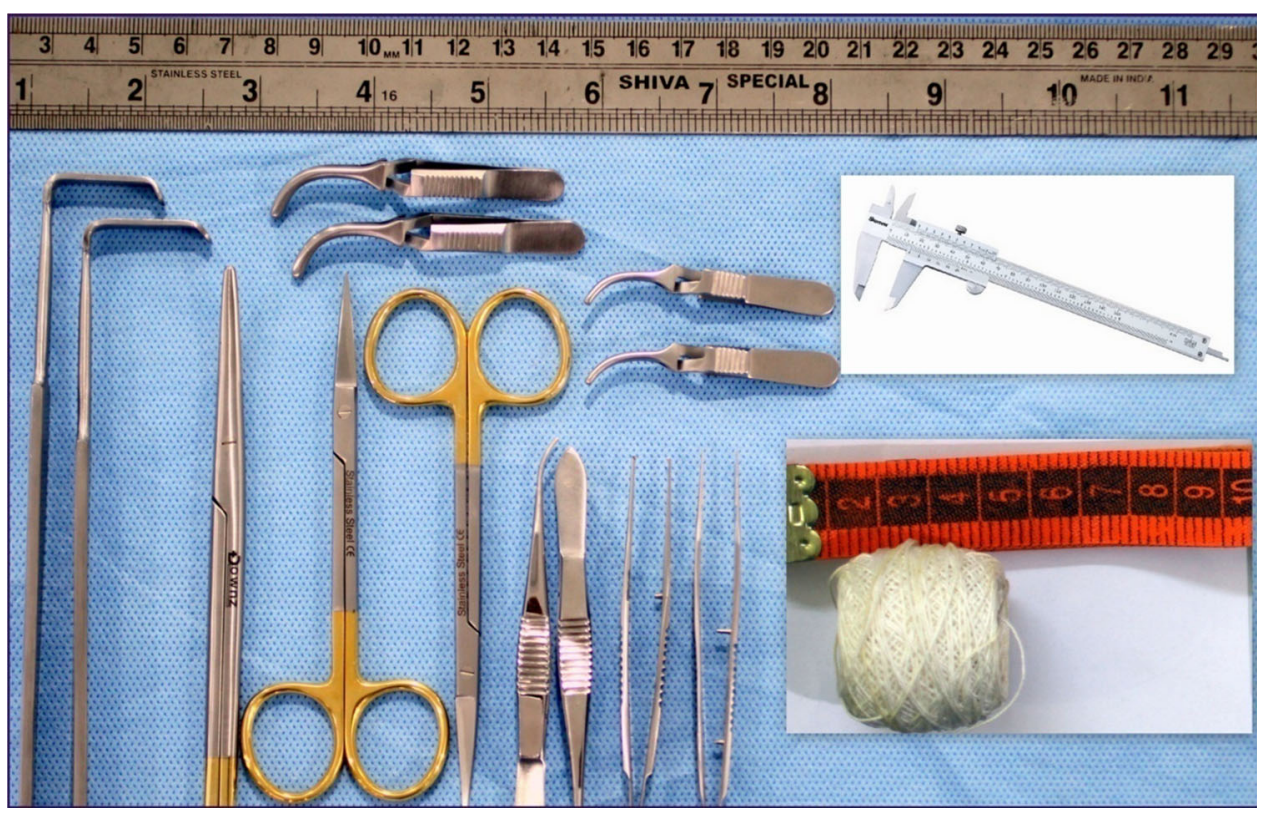

demise, hospitalization charts, and complications of delivery should be recorded.

\section{Cytogenetic Study}

Babies with malformations and very small fetuses where dysmorphic features may not be apparent should have chromosomal analysis. Samples are usually taken from the skin over the chest at the main incision site or the axilla using a sterile technique, cleansing the skin with sterile saline and not alcohol. Fascia, lungs, Achilles tendon, cartilage, and chorionic villous tissue are best suited for culture. In macerated fetuses, the placenta may be sampled.

\section{External Examination}

A complete systematic examination of the fetus/baby is performed using a standardized protocol, regardless of gestational age and proceeding in a cephalo-caudal direction (Fig. 7) in the Appendix. Fetal growth, presence of maceration, edema, dehydration, cyanosis, jaundice, dysmorphic features and injuries related to delivery, meconium staining are some of the features, looked for. External examination is summarized in Fig. 2 (anthropometry) and Fig. 3 (external examination).

\section{Incision}

The fetus is placed over a wedge-shaped autopsy block with hyperextended neck with shoulders and chest raised above the dissecting surface. A Y-shaped incision is usually employed such that the arms of the Y commence from the top of the shoulder, descend lateral and inferior to the nipples to meet in the mid-line at the sternal xiphoid process (Fig. 2). The vertical stem extends from the xiphoid, around the umbilicus to terminate at the symphysis pubis. The abdominal cavity is opened by the side of the umbilical vein, finger inserted under the umbilicus and the umbilical arteries, the urachus and the urinary bladder palpated. The course of the umbilical vein and the umbilical arteries is observed. The chest wall is exposed by reflecting the skin upwards and dissecting the subcutaneous and the muscle tissue.

\section{In Situ Examination and Evisceration}

Rokitansky method is an in situ examination of viscera, in part combined with en bloc removal. In the Virchow technique, the organs are removed one by one and dissected as removed. Letulle method is an en masse removal of thoracic, cervical, abdominal, and pelvic organs which are subsequently dissected into organ 
Fig. 2 i Head circumference (HC), ii chest circumference (CC), iii abdominal circumference, iv outer canthus (OC), v inner canthus (IC), vi interpupillary distance, vii ix wedge-shaped autopsy block, x Y-shaped incision philtrum length, viii foot length,
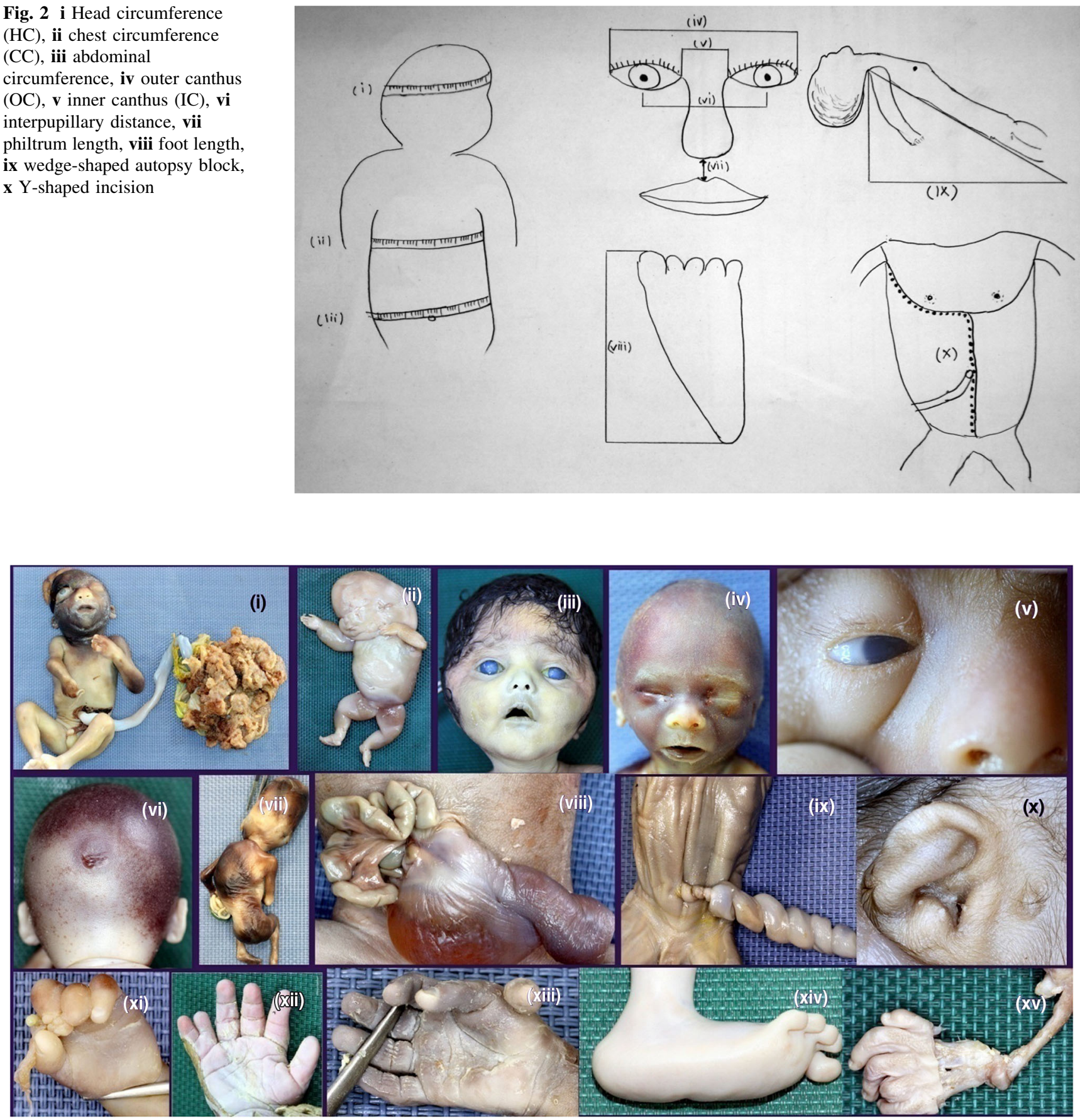

Fig. 3 i Amniotic band syndrome, ii nonimmune hydrops fetalis (Turner syndrome), iii blue sclera, iv microphthalmia, $\mathbf{v}$ Duane anomaly, vi occipital cephalocele, vii scoliosis, viii omphalocele, ix

blocks. An en bloc removal of viscera into functionallyrelated blocks (Gohn) is a compromise between the Virchow and en masse techniques. The choice of hypercoiled umbilical cord, $\mathbf{x}$ per-auricular skin tag, xi amputated fingers due to amniotic bands, xii post-axial polysyndactyly, xiii pre-

technique is made at the time of the autopsy by the prosector with the intent to salvage the maximum information possible (Figs. 4 and 5). axial polydactyly, $\mathbf{x i v}$ rocker bottom foot, $\mathbf{x v}$ radial aplasia 


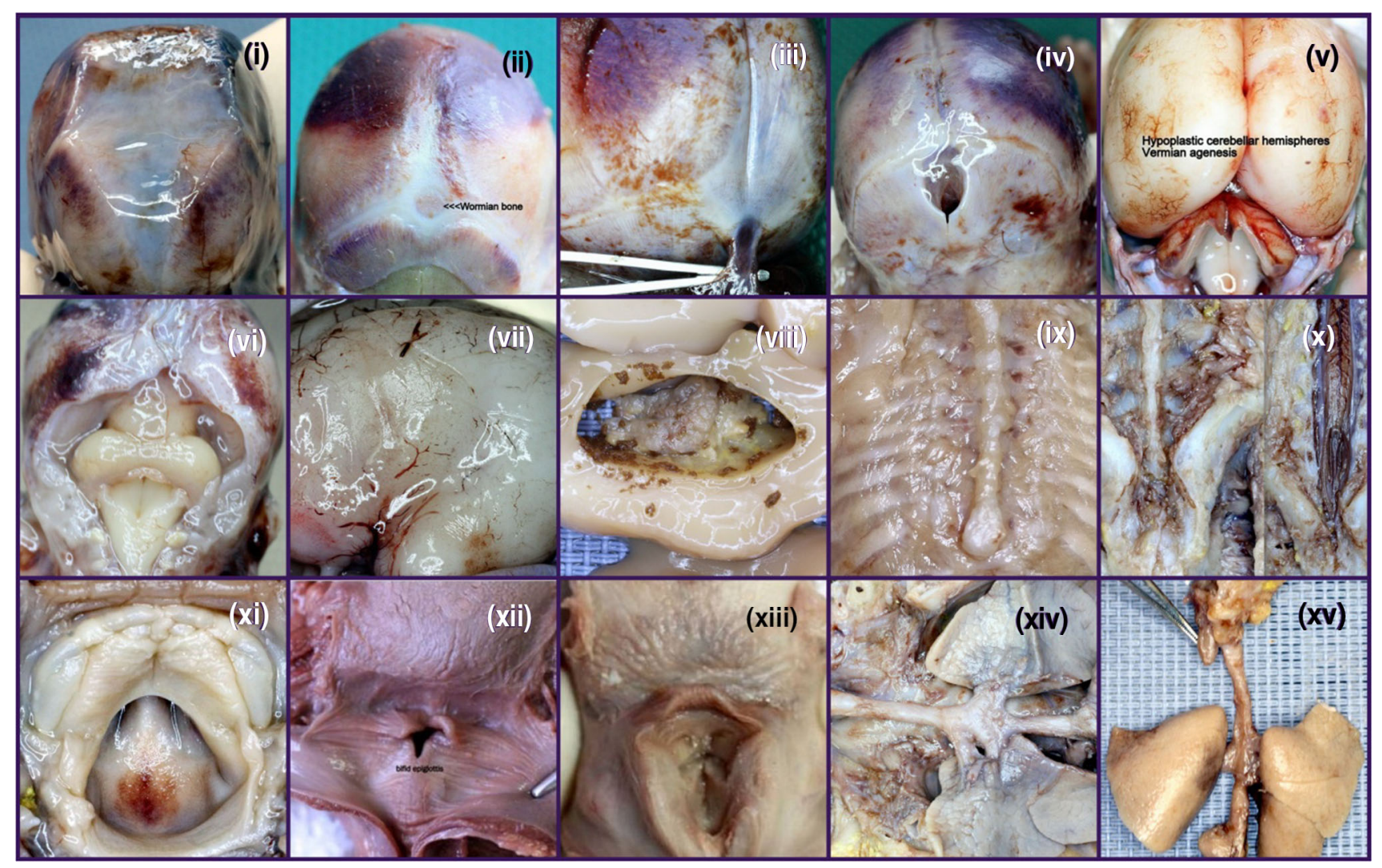

Fig. 4 i Wide anterior fontanel, ii wormian bone, iii and iv occipital cephalocle, $\mathbf{v}$ vermian agenesis, vi question mark dissection to display the brain stem, vii lissencephaly, viii ventriculomegaly with nodular

The abdominal organs are usually examined first commencing with recording of the situs. The position of appendix (which denotes the completion of mid-gut rotation), and the mesenteric attachment along with the position of ascending colon, transverse colon, descending colon, sigmoid colon, rectum, and anal canal inspected. The transverse colon can now be separated from the stomach allowing visual inspection of the spleen, splenic artery, and the pancreas. The descending colon can be retracted medially along with the peritoneum to visualize left kidney, left supra renal gland, and the left ureter. The presence of anomalies is documented. Uterus, ovaries, and fallopian tubes in the female and testes in the male are identified. The domes of the diaphragm are inspected. The liver, its visceral surface along with gall bladder, portahepatis, and the lesser omentum are examined. protrusions, ix caudal dysplasia sequence, $\mathbf{x}$ sacral agenesis (diabetes associated), xi cleft palate, xii bifid epiglottis, xiii congenital tracheal stenosis, $\mathbf{x i v}$ and $\mathbf{x v}$ tracheoesophageal fistula

The thoracic cavity is entered by first incising the sternoclavicular joints and then cutting the ribs $4 \mathrm{~mm}$ from the costochondral junction in an inverted $\mathrm{V}$ pattern. After examining the xiphoid process, the ribs are lifted off by grasping the xiphoid with toothed forceps. The external surface of the lungs and the lobations recorded. The heart and the great vessels are inspected next by removing the thymus and the pericardium. Absence of left brachiocephalic vein suggests persistent left superior vena cava. Cardiothoracic ratio is recorded and the thoracic situs determined. The position of the heart and the apex documented. The aorta, the great vessels, ductus arteriosus, and the pulmonary trunk and the pulmonary veins examined.

The heart is dissected in situ following a six step technique. Step 1: The right atrium is nicked at the lateral aspect, the incision extended cranially through the superior 


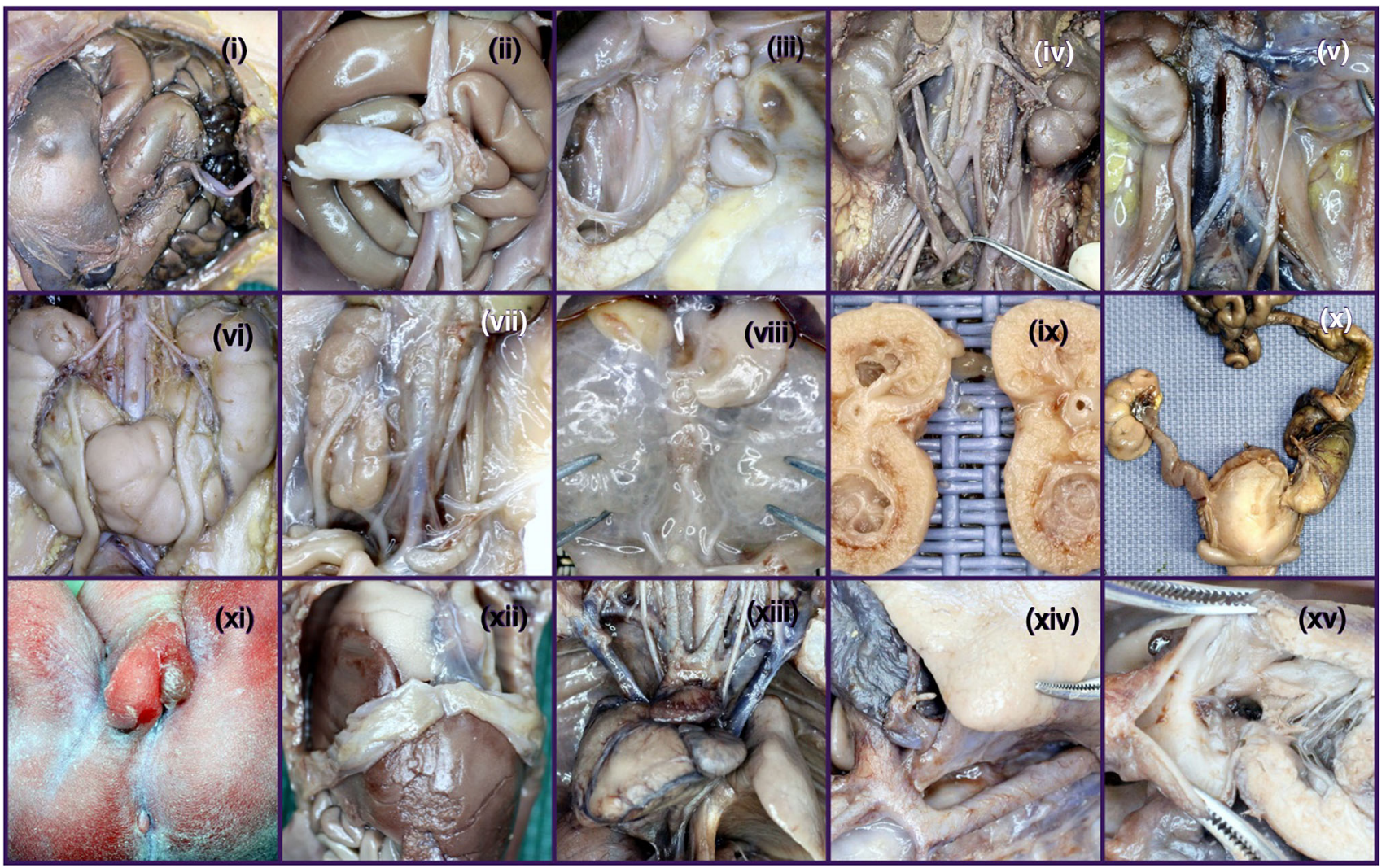

Fig. 5 i Aberrant umbilical vein, ii single umbilical artery, iii splenicules, iv double ureters, $\mathbf{v}$ ureteral dysplasia, vi horse shoe kidney, vii unilateral renal agenesis, viii polycystic kidney disease (autosomal recessive) with duplex ureters (right), ix renal cystic dysplasia, $\mathbf{x}$ Uro-Rectal-Septal-Malformation Sequence (URSMS) with vesico-uretero-rectal fistula, xi ambiguous genitalia with phallic like structure (URSMS), xii diaphragmatic hernia, xiii pulmonary agenesis (right), xiv right sided aorta, $\mathbf{x v}$ ventricular septal defect vena cava into the left brachiocephalic vein and caudally into the inferior vena cava to the diaphragm. Step 2: The right atrial wall is incised to the right ventricular apex $1 \mathrm{~cm}$ above the posterior descending coronary artery. Step 3: The right ventricular outflow tract is incised next from the apex, across the pulmonary valve and into the left pulmonary artery. Step 4: The left atrial appendage is nicked next with extension of the cut into the pulmonary veins. Step 5: The postero-lateral wall of the left ventricle is incised across the mitral valve to the apex. Step 6: Commencing from the apex, the anterior wall of the left ventricle, the aortic valve, ascending aorta and the aortic arch are cut next. At this juncture, the thoracic and the abdominal cavity are completely exposed and the evisceration of the organs can be done utilizing any of the aforementioned techniques.

The brain removal is facilitated by an incision over the cranium extending from behind one ear to the other. The conversion of this incision into a question mark posteriorly is useful for delineating the brainstem and herniations through the foramen magnum. After reflecting the skin, the length and the breadth of the fontanels are measured and the degree of tension noted. The skull is next opened by cutting along the suture lines commencing at the lateral corner of the anterior fontanel, thus producing frontal and parietal bone flaps. The frontal, parietal, and the occipital bones are further cut in an oval fashion such that a flap of bone is left at the lateral aspect. The brain is inspected in situ and so are falx and the tentorium. The falx is now cut along the sagittal sinus antero-posteriorly. The brain can be removed by cradling the skull and the brain in the palm of the left hand at the occiput and tilting the head backwards gently allowing the brain to dislodge from the calvarium. The cranial nerves are inspected and gently cut close to the bone and the stalk of the pituitary severed close 
to the brain. The tentorium is dissected around the periphery exposing the cerebellum. The cervical spinal column is transected as far into the foramen magnum as possible. The completely-disengaged brain is removed, weighed, inspected, and placed in formalin tank for 10-20 days for fixation. The brain can also be removed by using the same method under the water, a technique which eliminates parenchymal tear due to brain weight and gravity. Post removal of the brain, the base and the foramen magnum are inspected.

\section{Provisional Autopsy Diagnosis (PAD)}

The PAD usually is an extrapolation of gross external, internal, radiograph, frozen section, and Gram stain findings compiled within $24-48 \mathrm{~h}$ of the postmortem examination.

\section{Placental Examination}

The post mortem examination is incomplete without the placenta. Gross examination of the placenta is discussed in a separate write-up in this issue of the journal and is thus not included here.

\section{Microscopic Examination}

With the exception of malformations, the macroscopic appearance of fetal organs does not give specific information. Apart from identifying a cause or mechanism of death, microscopic examination can also help in assessment of gestational age.

\section{Final Diagnosis}

The final diagnosis is furnished only after the completion of microscopy. A summary of the case, comments, and pertinent references should be included in the final report.

\section{Classification of Perinatal Deaths}

The aim of classifying perinatal deaths is to derive strategies towards understanding the cause and ultimately prevent perinatal mortality. A good classification system integrates clinical factors, autopsy, and placental findings, takes into account the causative and contributory factors; thereby yielding a high percentage of classifiable causes. The ReCoDe system (Fig. 8) is a hierarchical classification that identifies the relevant condition at the time of death. It identifies what went wrong, not necessarily why [6]. The Tulip classification (Fig. 9) allows classification of underlying causes and mechanisms of perinatal mortality [7]. Both these systems have a low percentage of unexplained deaths (10-15\%). Classifying perinatal death is a complex process that requires a high level of expertise and is best accomplished at a well-organized, multidisciplinary, perinatal mortality meeting, where a conclusion is reached after discussion of all available clinical, pathological, and ancillary information.

Acknowledgments The author would like to thank the management of Fernandez Hospital and the entire staff of the Department of Perinatal Pathology, Fernandez Hospital for their support and recognize Srinivas V. for assisting with gross pictures.

\section{Compliance with Ethical Standards}

Conflict of interest None.

\section{Appendix}

See Figs. 6, 7, 8, and 9. 
FETAL / PERINATAL / NEONATAL DEATH POST MORTEM EXAMINATION CONSENT FORM

$\mathrm{I}$, (insert full name) give my consent for a complete / partial

(limited to ) autopsy examination of (my

fetus/baby/ward/relative) and I am the mother/father/competently authorized maternal or paternal relative of the deceased.

By giving my consent, I understand,

1. That, it may include removal, retention, macroscopic, microscopic examination or use of any of the body part/organ as deemed proper by the physicians.

2. That the autopsy is being done for academic reasons and not for medico legal purpose.

3. That any derived information may be utilized for diagnostic, therapeutic, scientific, research or publication purposes.

4. That the information would not be used for commercial or advertising purpose.

5. That my consent is only partially applicable for and inclusive of

\begin{tabular}{|l|l|l|l|}
\hline S. No & \multicolumn{1}{|c|}{ PROCEDURE } & \multicolumn{2}{|c|}{ CONSENT } \\
\cline { 3 - 4 } & & YES & NO \\
\hline I & Photography & & \\
\hline II & Radiological examination & & \\
\hline \hline III & External examination & & \\
\hline IV & Internal examination & & \\
\hline V & Removal of organs for examination purpose & & \\
\hline \hline VI & Retention of organs & & \\
\hline VII & Removal and examination of the brain and the spinal cord & & \\
\hline VIII & Mounting for academic purpose & & \\
\hline \hline IX & Publication & & \\
\hline
\end{tabular}

6. That I can revoke my consent any time prior to the autopsy. However once the autopsy has been performed by the physicians, it will not be possible to revoke the consent.

7. That if the information is utilized for publication purpose, every attempt would be made to ensure anonymity.

8. That I would have no objection to the text of the article and its publication and distribution.

9. That the consent form and information contained in the consent form as well as procedures pertaining to the autopsy have been explained to me in a language that I can understand.

10. That once the autopsy is completed I wish,

\begin{tabular}{|l|l|l|}
\hline The baby to be returned to the family & & \\
\hline \hline The baby to be sent to the burial ground for the performance of the final rites & & \\
\hline The baby be retained by the institute for academic purpose & & \\
\hline
\end{tabular}

Consenter's Signature:

Physician's signature:

Date:

Fig. 6 Perinatal autopsy consent form template used at the institute 


\begin{tabular}{|c|c|}
\hline A. Fetus & 1. Lethal congenital anomaly \\
\hline & $\begin{array}{l}\text { 2. Infection } \\
\qquad 2.1 \text { Chronic-e.g. TORCH } \\
\qquad 2.2 \text { Acute } \\
\text { 3. Non-immune hydrops } \\
\text { 4. Iso-immunisation } \\
\text { 5. Fetomaternal haemorrhage } \\
\text { 6. Twin-twin transfusion } \\
\text { 7. Fetal growth restriction } \\
\text { 8. Other }\end{array}$ \\
\hline B. Umbilical Cord & 1. Prolapse \\
\hline & $\begin{array}{l}\text { 2. Constricting loop or knot }{ }^{2} \\
\text { 3. Velamentous insertion } \\
\text { 4. Other }\end{array}$ \\
\hline C. Placenta & 1. Abruptio \\
\hline & $\begin{array}{l}\text { 2. Praevia } \\
\text { 3. Vasa Praevia } \\
\text { 4. Placental insufficiency /infarction }{ }^{3} \\
\text { 5. Other }\end{array}$ \\
\hline D. Amniotic fluid & $\begin{array}{l}\text { 1. Chorioamnionitis } \\
\text { 2. Oligohydramn ios }{ }^{2} \\
\text { 3. Polyhydramnios }{ }^{2} \\
\text { 4. Other }\end{array}$ \\
\hline E. Uterus & $\begin{array}{l}\text { 1. Rupture } \\
\text { 2. Other }\end{array}$ \\
\hline F. Mother & 1. Diabetes \\
\hline - & $\begin{array}{l}\text { 2. Thyroid diseases } \\
\text { 3. Essential Hypertension } \\
\text { 4. Hypertensive diseases in pregnancy } \\
\text { 5. Lupus/Antiphospholipid Syndrome } \\
\text { 6. Cholestasis } \\
\text { 7. Drug abuse } \\
\text { 8. Other }\end{array}$ \\
\hline G. Intrapartum & $\begin{array}{l}\text { 1. Asphyxia } \\
\text { 2. Birth Trauma }\end{array}$ \\
\hline H. Trauma & $\begin{array}{l}\text { 1. External } \\
\text { 2. Iatrogenic }\end{array}$ \\
\hline I. Unclassified & $\begin{array}{l}\text { 1. No relevant condition identified } \\
\text { 2. No information available }\end{array}$ \\
\hline
\end{tabular}

\section{$\mathrm{ReCoDe}$}

\section{Classification of stillbirth by Relevant Condition at Death}

This system seeks to identify the condition(s) which existed at the time of death in-utero. The classification is based on the following principles:

1. Stillbirths are distinct from neonatal deaths and warrant their own classification.

2. There is hence no need for a subclassification according to gestation, as 'prematurity' is not a relevant cause or condition for stillbirths.

3. There is no subclassification according to weight, but one related to fetal growth status, based on weight-for-gestation.

4. The classification emphasises what went wrong, not necessarily 'why'. Hence, more than one category can be coded.

5. The hierarchy starts from conditions affecting the fetus and moves outwards, in simple anatomical categories (A-F) which are subdivided into pathophysiological conditions.

6. The primary condition should be the highest on the list that is applicable to a case.

Footnotes in table:

1. Defined as $<10$ th customised weight-for-gestation percentile (centile calculator is available at www.gestation.net/centile)

2. If severe enough to be considered relevant

3. Histological diagnosis

www.perinatal.nhs.uk/recode

Fig. 8 ReCoDe classification 


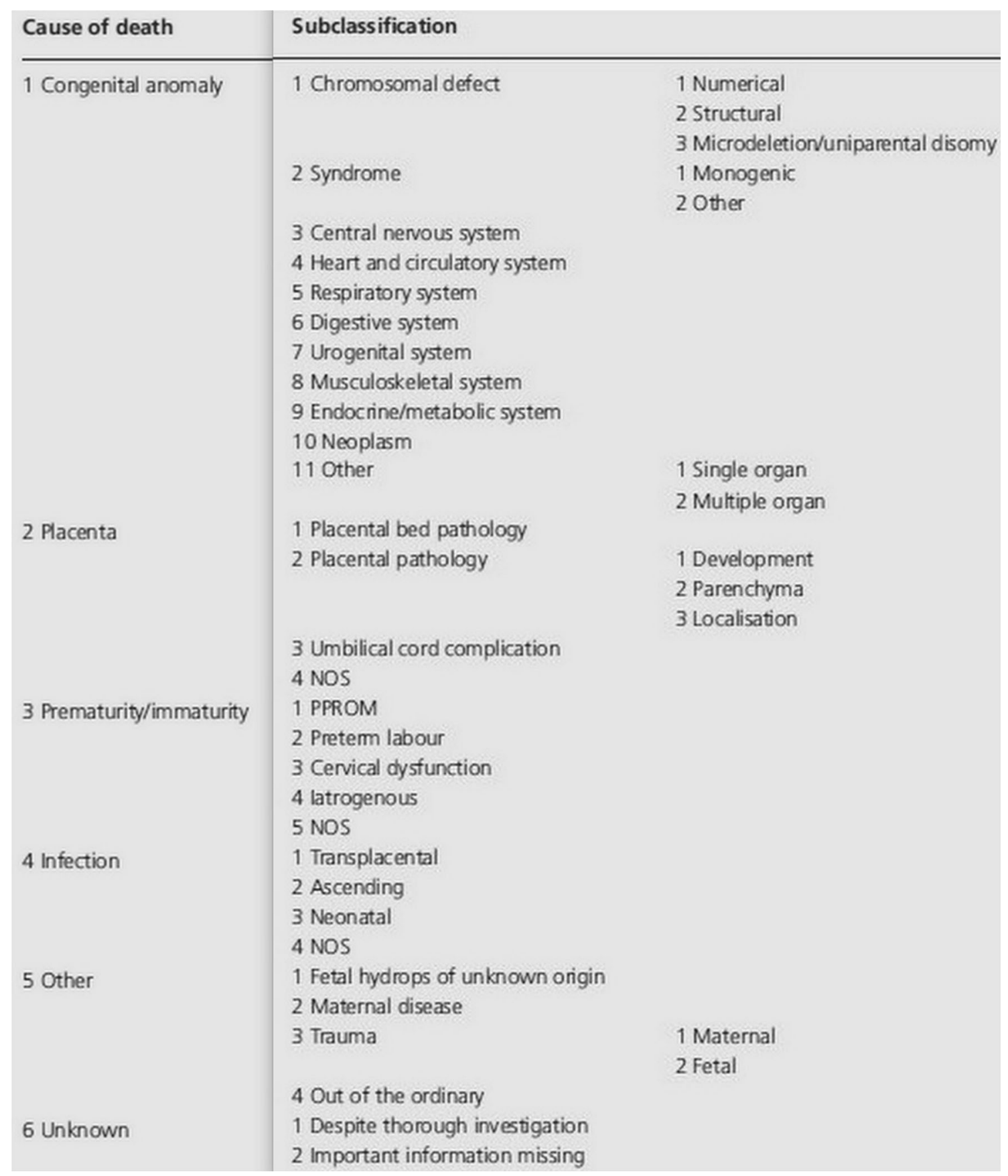

Fig. 9 Tulip classification

\section{References}

1. College of American Pathologists Pamphlet. Autopsy: aiding the living by understanding death. Northfield: College of American Pathologist (CAP); 2001.

2. Gilbert-Barness E, Kapur R, Oligny LL, Siebert J, editors. Potter's pathology of the fetus, infant and child. St. Louis, MO: Mosby Elsevier; 2007.

3. Stocker JT, Dehner LP, Husain AN, editors. Stocker and Dehner's pediatric pathology. Philadelphia, PA: Lippincott Williams and Wilkins; 2011.
4. Wigglesworth JS, Singer DB, editors. Textbook of fetal and perinatal pathology. Oxford: Blackwell Scientific Publications; 1991.

5. Cohen MC, Scheimberg I, editors. The pediatric and perinatal autopsy manual. Cambridge: Cambridge University Press; 2014.

6. Gardosi J, Kady SM, McGeown P, Francis A, Tonks A. Classification of stillbirth by relevant condition at death $(\mathrm{ReCoDe})$ : population based cohort study. BMJ. 2005;331(7525):1113-7.

7. Korteweg FJ, Gordijn SJ, Timmer A, Erwich JJ, Bergman KA, Bouman $\mathrm{K}$, et al. The Tulip classification of perinatal mortality: introduction and multidisciplinary inter-rater agreement. BJOG. 2006;113(4):393-401. 